\title{
COMPUTATIONALLY EFFICIENT APPROXIMATIONS OF THE JOINT SPECTRAL RADIUS
}

\author{
VINCENT D. BLONDEL AND YURII NESTEROV
}

\begin{abstract}
The joint spectral radius of a set of matrices is a measure of the maximal asymptotic growth rate that can be obtained by forming long products of matrices taken from the set. This quantity appears in a number of application contexts but is notoriously difficult to compute and to approximate. We introduce in this paper a procedure for approximating the joint spectral radius of a finite set of matrices with arbitrary high accuracy. Our approximation procedure is polynomial in the size of the matrices once the number of matrices and the desired accuracy are fixed.

For the special case of matrices with non-negative entries we give elementary proofs of simple inequalities that we then use to obtain approximations of arbitrary high accuracy. From these inequalities it follows that the spectral radius of matrices with non-negative entries is given by the simple expression

$$
\rho\left(A_{1}, \ldots, A_{m}\right)=\lim _{k \rightarrow \infty} \rho^{1 / k}\left(A_{1}^{\otimes k}+\cdots+A_{m}^{\otimes k}\right)
$$

where it is somewhat surprising to notice that the right hand side does not directly involve any mixed product between the matrices $\left(A^{\otimes k}\right.$ denotes the $k$-th Kronecker power of $A$ ).

For matrices with arbitrary entries (not necessarily non-negative) we introduce an approximation procedure based on semi-definite liftings that can be implemented in a recursive way. For two matrices, even the first step of the procedure gives an approximation whose relative accuracy is at least $1 / \sqrt{2}$, that is, more than $70 \%$. The subsequent steps improve the accuracy but also increase the dimension of the auxiliary problems from which the approximation can be found.

Our approximation procedures provide approximations of relative accuracy $1-\epsilon$ in time polynomial in $n^{(\ln m) / \epsilon}$, where $m$ is the number of matrices and $n$ is their size. These bounds are close from optimality since we show that, unless $\mathrm{P}=\mathrm{NP}$, no approximation algorithm is possible that provides a relative accuracy of $1-\epsilon$ and runs in time polynomial in $n$ and $1 / \epsilon$.

As a by-product of our results we prove that a widely used approximation of the joint spectral radius based on common quadratic Lyapunov functions (or on ellipsoid norms) has relative accuracy $1 / \sqrt{m}$, where $m$ is the number of matrices. ${ }^{1}$
\end{abstract}

\footnotetext{
Date: February 1, 2008.

${ }^{1}$ This paper presents research results of the Belgian Program on Interuniversity Poles of Attraction initiated by the Belgian State, Prime Minister's Office, Science Policy Programming. The scientific responsibility is assumed by the authors.
} 


\section{INTRODUCTION}

Let $\|\cdot\|$ be a matrix norm. The spectral radius of the real matrix $A$ is defined by

$$
\rho(A)=\lim _{k \rightarrow+\infty}\left\|A^{k}\right\|^{1 / k} .
$$

The spectral radius of a matrix does not depend on the chosen matrix norm and it is also equal to $\rho(A)=\max \{|\lambda|: \lambda$ is an eigenvalue of $A\}$ (see for example [22, Corollary 5.6.14]). The definition of spectral radius can be extended to sets of matrices in a natural way. The joint spectral radius of a set of matrices is a quantity introduced by Rota and Strang in the early 60's that measures the maximal asymptotic growth rate that can be obtained by forming long products of matrices; see [29]. The general definition is for arbitrary sets of matrices but we shall consider here only finite sets. Let $\left\{A_{1}, \ldots, A_{m}\right\}$ be some set of real matrices. To the finite sequence $\sigma=\left(\sigma_{1}, \sigma_{2}, \ldots, \sigma_{k}\right) \in\{1, \ldots, m\}^{k}$ we associate the corresponding matrix product

$$
A_{\sigma}=A_{\sigma_{k}} \cdots A_{\sigma_{2}} A_{\sigma_{1}} .
$$

With this notation, the joint spectral radius is defined by

$$
\rho\left(A_{1}, \ldots, A_{m}\right)=\limsup _{k \rightarrow+\infty} \max _{\sigma \in\{1, \ldots, m\}^{k}}\left\|A_{\sigma}\right\|^{1 / k} .
$$

As for the single-matrix case, the joint spectral radius does not depend on the matrix norm used. To see this, remember that any two matrix norms $\|\cdot\|_{1}$ and $\|\cdot\|_{2}$ are related by $\alpha\|A\|_{1} \leq\|A\|_{2} \leq \beta\|A\|_{1}$ for some $0<\alpha<\beta$. For any product $\sigma \in\{1, \ldots, m\}^{k}$ one has $\alpha^{1 / k}\left\|A_{\sigma}\right\|_{1}^{1 / k} \leq\left\|A_{\sigma}\right\|_{2}^{1 / k} \leq \beta^{1 / k}\left\|A_{\sigma}\right\|_{1}^{1 / k}$ and by letting $k$ tend to infinity we conclude that the joint spectral radius is well defined independently of the matrix norm used.

A definition analogous to (1.2) is possible by replacing the norm appearing in the definition by a spectral radius. The quantity defined in this way is the generalized spectral radius introduced in [14. In [3], the joint and generalized spectral radii of finite (or bounded) sets of matrices are proved to be equal (see also [16, Theorem 1] for an elementary proof); thus reinforcing the status of the joint spectral radius as a legitimate generalization of the spectral radius of a single matrix. In the sequel we shall deal only with the spectral radius defined with a norm, as in (1.2).

Since its introduction in the 60's the joint spectral radius has appeared in a number of different contexts; see, e.g., [31] or [15] for recent short surveys ${ }^{2}$. Let us illustrate one application in a dynamical system context. Consider the simple discrete-time linear inclusion

$$
x_{k+1} \in\left\{A_{1}, \ldots, A_{m}\right\} x_{k}, \quad x_{0} \in \mathbf{R}^{n}
$$

in which at each step a particular linear transformation is chosen from of a finite number of possible choices. The maximal asymptotic rate of growth of the trajectories associated to such a system is given by the joint spectral radius $\rho\left(A_{1}, \ldots, A_{m}\right)$. (In the discrete linear inclusion literature, the logarithm of the joint spectral radius is sometimes called

\footnotetext{
${ }^{2}$ Google returns 625 entries upon entry of the query "joint spectral radius".
} 
Lyapunov indicator, see for example [2.) In particular, all possible trajectories will converge to the origin if and only if $\rho\left(A_{1}, \ldots, A_{m}\right)<1$. The joint spectral radius can thus be associated with the stability properties of time-varying linear systems in the worst case over all possible time variations. It also occur in the context of "asynchronous" [33] or "desynchronised" 24] systems. Besides systems-theoretic interpretations, the concept is pervasive in many areas of applied mathematics such as in wavelets [14, iterated function systems, random walks, fractals, numerical solutions to ordinary differential equations [18, discrete-event systems [10], interpolation [38, and coding theory [28].

Despite its natural interpretation, the joint spectral radius is difficult to compute. Questions related to its computability and to the existence of efficient approximation algorithms have been posed more than a decade ago (see [34] and 25]). In principle, the spectral radius can be approximated to any desired accuracy by computing converging sequences of upper and lower bounds. The following bounds, proved in [25],

$$
\max _{\sigma \in\{1, \ldots, m\}^{k}} \rho\left(A_{\sigma}\right)^{1 / k} \leq \rho\left(A_{1}, \ldots, A_{m}\right) \leq \max _{\sigma \in\{1, \ldots, m\}^{k}}\left\|A_{\sigma}\right\|^{1 / k}
$$

can be evaluated for increasing values of $k$ and lead to arbitrary accurate approximations of $\rho$ (see, e.g., [15] or [17]). Such approximation algorithms can in turn be used in procedures that decide, after finitely many steps, whether $\rho>1$ or $\rho<1$ (such procedures are given, e.g., by Brayton and Tong [13] in a system theory context and by Barabanov [2] in the context of discrete linear inclusions). These procedures may however not terminate when $\rho$ happens to be equal to 1 and the existence of algorithms for computing arbitrarily precise approximations of $\rho$ does therefore not rule out the possibility that the decision problem " $\rho<1$ ?" is undecidable. It is so far unknown whether this is the case or not (see 25] for a discussion of this issue and for a description of its connection with the finiteness conjecture that has since then been proved to be false; see [11] and [4]). A negative result in this direction is given in [8] where it is proved that the related problem " $\rho \leq 1$ ?" is algorithmically undecidable.

Approximations of the joint spectral radius that are directly based on the inequalities (1.3) are expensive to compute. In [27], the exponential number of products that appear in the direct and naive computation of the bounds in (1.3) is reduced by avoiding duplicate computation of cyclic permutations; the total number of product to consider remains however exponential. In addition to this, there are to this date no known theoretical guarantees for the rate of convergence of the bounds appearing in (1.3). Approximations of arbitrary degree of accuracy can be computed, but at a price that may happen to be prohibitive.

There are in fact intrinsic limitations for the rate at which the joint spectral radius can be approximated. Let us say that the value $\xi$ approximates the value $\rho$ with relative accuracy $\mu \in[0,1]$ (or $100 \mu \%$ ), if $\mu \xi \leq \rho \leq \xi$. By using a small adaptation of a proof 
appearing in 32 we show that, unless $\mathrm{P}=\mathrm{NP}$, there is no algorithm that can compute the joint spectral radius of two matrices with relative accuracy $1-\epsilon$ in time polynomial in the size of the matrices and in $1 / \epsilon$ (see later for more precise definitions). Despite this negative result it is still conceivable, as pointed in [9], that for any fixed desired accuracy, there exists a polynomial time algorithm that computes the joint spectral radius of the matrices with that accuracy. We prove in this paper that this is indeed the case. In Theorem [5. we prove that the joint spectral radius of $m$ matrices of size $n$ can be approximated with relative accuracy $1 / \sqrt{m}$ by computing the spectral radius of a single matrix whose size is less than $n^{2}$. This procedure can be applied in a recursive way and in general we show how a relative accuracy of $(1 / \sqrt{m})^{1 / k}$ can be obtained by computing the spectral radius of a single matrix of size less than $n^{2 k}$. As an illustration, the spectral radius of two matrices of size $n$ can be computed with an accuracy of $70 \%$ by computing the spectral radius of a single matrix of size $n^{2}$, and an accuracy of $95 \%$ can be obtained for the joint spectral radius of three matrices by computing the spectral radius of a single matrix of size $n^{11}$. More generally, for any number of matrices in the set and desired relative accuracy we construct in Section 4 a single matrix whose spectral radius approximates the joint spectral radius with the desired accuracy. The approximation procedure runs in polynomial time once the desired accuracy and the number of matrices in the set are fixed. More precisely, our approximation procedures provide approximations of relative accuracy $1-\epsilon$ in time polynomial in $n^{(\ln m) / \epsilon}$, where $m$ is the number of matrices and $n$ is their size. Notice that $n^{(\ln m) / \epsilon}=e^{(\ln n \ln m) / \epsilon}=m^{(\ln n) / \epsilon}$ and so our approximation procedure also runs in time polynomial in the number of matrices once the desired accuracy and the size of the matrices are fixed. These bounds are close from optimality since we prove that, unless $\mathrm{P}=\mathrm{NP}$, no approximation polynomial time algorithm in $n$ and $1 / \epsilon$ is possible.

We now briefly describe how our results are obtained and how the paper is organized. We first notice in Section 2 that for matrices with non-negative entries the joint spectral radius satisfies

$$
\frac{1}{m} \rho\left(A_{1}+\cdots+A_{m}\right) \leq \rho\left(A_{1}, \ldots, A_{m}\right) \leq \rho\left(A_{1}+\cdots+A_{m}\right)
$$

(The left-hand side inequality is valid for arbitrary matrices; there is no need to assume that the matrices have non-negative entries.) Matrices with non-negative entries are exactly those matrices $A$ that are such that $A \mathbf{R}_{+}^{n} \subseteq \mathbf{R}_{+}^{n}$ and we prove in Theorem 1 that the inequalities (1.4) are satisfied not only for sets of matrices with non-negative entries but also for sets of matrices that leave a proper cone invariant, i.e., matrices $A_{i}$ that are such that $A_{i} K \subseteq K$ for some proper cone $K$ and all $i$. Thus for these matrices the inequalities (1.4) provide a relative accuracy $1 / m$. This accuracy can be improved by considering Kronecker powers of matrices. In Section 3 we give an elementary proof that the joint spectral radius of the $k$-th Kronecker powers of the matrices in a set is equal to the $k$-th power of the joint spectral radius of the set (Theorem 3). Combining this with 
the relation (1.4) we prove that the approximation

$$
\rho^{1 / k}\left(A_{1}^{\otimes k}+\cdots+A_{m}^{\otimes k}\right)
$$

has relative accuracy $1 / m^{1 / k}$. Some of the arguments used to derive this result are valid only for matrices that have a common proper invariant cone. In Section 4 we show how similar arguments can be used for arbitrary matrices. We introduce a semi-definite lifting procedure that transforms a linear operator acting $\mathbf{R}^{n}$ into a linear operator acting on the space of symmetric $n \times n$ matrices. It appears that under this transformation the joint spectral radius is simply squared. Moreover, the lifting has the interesting feature that the operator defined in this way leaves the cone of semi-definite matrices invariant. This observation leads to a simple approximation procedure (Theorem 5) for arbitrary matrices. Implementation issues and numerical examples are provided in Section 5. Our approximation procedure provides a relative accuracy $1-\epsilon$ in time polynomial in $n^{(\ln m) / \epsilon}$. We prove in Section 6] (Theorem 6) that the same accuracy cannot be obtained in time polynomial in $n$ and $1 / \epsilon$ unless $\mathrm{P}=\mathrm{NP}$. The semi-definite lifting introduced in Section 4 is a fundamental and powerful tool for stability analysis. As an illustration of this, we prove in Section 7 how to apply our results to analyze the quality of the so-called ellipsoid approximation of the joint spectral radius. We prove that the ellipsoid approximation (a notion that is formally defined in [4 but that is implicitly present in a number of earlier contributions on hybrid and time-varying systems, see, e.g., 21] and [1] as well as some of the references in [26]) is an approximation of guaranteed accuracy $1 / \sqrt{m}$; a proof of this corollary can also be extracted from Section 3 of [21]. For matrices of high dimension this result significantly improves the earlier bound of $1 / \sqrt{n}$ proved in [4] and in [1]. Finally, in a last section, we discuss some of our results.

\section{Approximation For MATRICES LEAVING A CONE INVARIANT}

In this section, we consider sets of matrices that leave a proper cone invariant and we show with elementary arguments how joint spectral radius approximations of guaranteed accuracy can easily be computed for this case. We start with a proof that the spectral radius of a convex combination of matrices is always less or equal to the joint spectral radius of the matrices. This result is valid for all sets of matrices (there is no need to assume that the matrices have non-negative entries) and is proved in [6] using the main result of [24]. Here we present a direct and elementary justification.

Lemma 1. For any set of matrices $\left\{A_{i}: i=1, \ldots, m\right\}$ and any $\alpha_{i} \geq 0$ satisfying $\sum_{i=1}^{n} \alpha_{i}=1$ we have:

$$
\rho\left(\sum_{i=1}^{m} \alpha_{i} A_{i}\right) \leq \rho\left(A_{1}, \ldots, A_{m}\right) .
$$


Proof. Let us fix some $\alpha_{i} \geq 0$ with $\sum_{i} \alpha_{i}=1$ and an integer $k \geq 1$. Then

$$
\left\|\left(\sum_{i=1}^{m} \alpha_{i} A_{i}\right)^{k}\right\|=\left\|\sum_{\sigma \in\{1, \ldots, m\}^{k}} \alpha_{\sigma} A_{\sigma}\right\| \leq \sum_{\sigma \in\{1, \ldots, m\}^{k}} \alpha_{\sigma}\left\|A_{\sigma}\right\| \leq \max _{\sigma \in\{1, \ldots, m\}^{k}}\left\|A_{\sigma}\right\|
$$

but then also

$$
\lim _{k \rightarrow \infty}\left\|\left(\sum_{i=1}^{m} \alpha_{i} A_{i}\right)^{k}\right\|^{1 / k}=\limsup _{k \rightarrow \infty}\left\|\left(\sum_{i=1}^{m} \alpha_{i} A_{i}\right)^{k}\right\|^{1 / k} \leq \limsup _{k \rightarrow \infty} \max _{\sigma \in\{1, \ldots, m\}^{k}}\left\|A_{\sigma}\right\|^{1 / k}
$$

and the result then follows from the definitions of the spectral radius (1.1) and of the joint spectral radius (1.2).

An immediate corollary is given by:

\section{Corollary 1.}

$$
\frac{1}{m} \rho\left(\sum_{i=1}^{m} A_{i}\right) \leq \rho\left(A_{1}, \ldots, A_{m}\right)
$$

The example $A_{1}=A, A_{2}=-A$ clearly shows that we cannot hope in general to have $\rho\left(A_{1}, \ldots, A_{m}\right) \leq \rho\left(\sum_{i=1}^{m} A_{i}\right)$. This inequality is nevertheless satisfied when the matrices $A_{i}$ leave a proper cone invariant. A cone in $\mathbf{R}^{n}$ is a subset $K \subseteq \mathbf{R}^{n}$ such that $\lambda v \in K$ for all $\lambda \geq 0$ and $v \in K$. We say that a cone $K$ is proper if it is closed, convex, has nonempty interior, and contains no straight line. For example, the set of vectors with non-negative entries $\mathbf{R}_{+}^{n}$ is a proper cone but $\mathbf{R}^{n}$ itself is not (see 30, for more background on cones and proper cones). We shall say that the matrices $A_{i}$ leave a proper cone invariant if there exists a proper cone $K \subseteq \mathbf{R}^{n}$ such that $A_{i} K \subseteq K$ for all $i$. For example, matrices with non-negative entries leave the proper cone $\mathbf{R}_{+}^{n}$ invariant.

Lemma 2. Associated to any proper cone $K$ there is a matrix norm $\|\cdot\|_{K}$ that satisfies $\|A\|_{K} \leq\|A+B\|_{K}$ for all matrices $A$ and $B$ that leave the cone $K$ invariant.

Proof. Most usual matrix norms satisfy this property when $K=\mathbf{R}_{+}^{n}$; we provide a construction for arbitrary proper cones $K$.

Let $\|\cdot\|$ be some arbitrary vector norm. The dual vector norm $\|\cdot\|_{*}$ is defined by $\|w\|_{*}=\max _{\|v\|=1} w^{T} v$. Assume that $K$ is a proper cone such that $A_{i} K \subseteq K$. Define the dual of $K$ by

$$
K^{*}=\left\{w \in \mathbf{R}^{n}: w^{T} v \geq 0 \text { for all } v \in K\right\} .
$$

The dual of a proper cone is again a proper cone, see [30]. Let us now consider the quantity

$$
\|A\|_{K}=\max _{\substack{v \in K, w \in K^{*} \\\|v\|=\|w\|_{*}=1}} w^{T} A v
$$


It is easy to verify that because $K$ and its dual are proper cones, the quantity $\|\cdot\|_{K}$ is indeed a matrix norm. Moreover, by the definition of the norm, matrices $A$ and $B$ that satisfy $A K \subseteq K$ and $B K \subseteq K$, are such that $\|A\|_{K} \leq\|A+B\|_{K}$.

We may now state the result of this section.

Theorem 1. Let $\left\{A_{i}: i=1, \ldots, m\right\}$ be a set of matrices that leave a proper cone invariant. Then

$$
\frac{1}{m} \rho\left(\sum_{i=1}^{m} A_{i}\right) \leq \rho\left(A_{1}, \ldots, A_{m}\right) \leq \rho\left(\sum_{i=1}^{m} A_{i}\right)
$$

Proof. The lower bound in (2.2) is already established in Corollary 1. Assume that the matrices in $\left\{A_{i}: i=1, \ldots, m\right\}$ leave the proper cone $K$ invariant and define the matrix norm $\|\cdot\|_{K}$ as in Lemma 2. Since $A_{i} K \subseteq K$, we also have $A_{\sigma} K \subseteq K$ for all $\sigma \in\{1, \ldots, m\}^{k}$ but then also

$$
\max _{\sigma \in\{1, \ldots, m\}^{k}}\left\|A_{\sigma}\right\|_{K} \leq\left\|\sum_{\sigma \in\{1, \ldots, m\}^{k}} A_{\sigma}\right\|_{K}=\left\|\left(\sum_{i=1}^{m} A_{i}\right)^{k}\right\|_{K}
$$

and therefore

$$
\rho\left(A_{1}, \ldots, A_{m}\right)=\limsup _{k \rightarrow \infty} \max _{\sigma \in\{1, \ldots, m\}^{k}}\left\|A_{\sigma}\right\|_{K}^{1 / k} \leq \limsup _{k \rightarrow \infty}\left\|\left(\sum_{i=1}^{m} A_{i}\right)^{k}\right\|_{K}^{1 / k}=\rho\left(\sum_{i=1}^{m} A_{i}\right) .
$$

It is interesting to notice that the conclusion of Theorem 1 does not directly involve the cone $K$. The existence of a proper cone satisfying the hypotheses suffices to conclude and the exact nature of the cone is irrelevant.

A matrix with non-negative entries leaves the cone $\mathbf{R}_{+}^{n}$ invariant and so we have the following corollary.

Corollary 2. Let the matrices $A_{i}, i=1, \ldots, m$, have non-negative entries. Then

$$
\frac{1}{m} \rho\left(\sum_{i=1}^{m} A_{i}\right) \leq \rho\left(A_{1}, \ldots, A_{m}\right) \leq \rho\left(\sum_{i=1}^{m} A_{i}\right)
$$

\section{KRONECKER LIFTING}

We now describe a way to exploit Theorem 1 for obtaining approximations of arbitrary high accuracy. Our approximations involve Kronecker powers of matrices. The Kronecker product of two matrices $A \in R^{p_{1} \times q_{1}}$ and $B \in R^{p_{2} \times q_{2}}$ is the $p_{1} p_{2} \times q_{1} q_{2}$ matrix defined by

$$
A \otimes B=\left(\begin{array}{ccc}
A_{1,1} B & \ldots & A_{1, q_{1}} B \\
& \ldots & \\
A_{p_{1}, 1} B & \ldots & A_{p_{1}, q_{1}} B
\end{array}\right) .
$$


We also define the Kroneker power of a matrix:

$$
A^{\otimes k}=\underbrace{A \otimes A \ldots A \otimes A}_{k \text { times }} .
$$

There is no need for parenthesis in this expression since the Kronecker product of two matrices is an associative operation. Let us prove some elementary properties.

Lemma 3. For matrices of appropriate sizes we have:

(1) $(A \otimes B)^{T}=A^{T} \otimes B^{T}$;

(2) $\left(A_{1} \otimes B_{1}\right)\left(A_{2} \otimes B_{2}\right)=\left(A_{1} A_{2}\right) \otimes\left(B_{1} B_{2}\right)$;

(3) $\left(A^{\otimes k}\right)^{T}=\left(A^{T}\right)^{\otimes k}$;

(4) $A^{\otimes k} B^{\otimes k}=(A B)^{\otimes k}$;

(5) Let $\|\cdot\|$ denote the spectral matrix norm induced by the standard Euclidean vector norm. Then $\left\|A^{\otimes k}\right\|=\|A\|^{k}$.

Proof. The first statement directly follows from the definition of the Kronecker product. The second statement is quite standard; see, e.g., Section 4.2 in 22 . The third and fourth statement follow, respectively, from repeated application of the first and second statement. Finally, for proving the last statement, note that by using (3) and (4) we obtain

$$
\left\|A^{\otimes k}\right\|^{2}=\max _{\|x\|=1} x^{T}\left(A^{\otimes k}\right)^{T} A^{\otimes k} x=\max _{\|x\|=1} x^{T}\left(A^{T}\right)^{\otimes k} A^{\otimes k} x=\max _{\|x\|=1} x^{T}\left(A^{T} A\right)^{\otimes k} x .
$$

The matrix $\left(A^{T} A\right)^{\otimes k}$ is symmetric and so the last expression is also equal to the largest magnitude of the eigenvalues of $\left(A^{T} A\right)^{\otimes k}$. For a matrix $B$ the eigenvalues of the matrix $B^{\otimes k}$ are given by all possible products of $k$ eigenvalues of $B$ (see Theorem 4.2.12 in [22]) and we therefore conclude $\max _{\|x\|=1} x^{T}\left(A^{T} A\right)^{\otimes k} x=\|A\|^{k}$.

We can now prove a useful identity for the spectral radius of Kronecker powers of matrices.

Theorem 2. Let $\left\{A_{i}: i=1, \ldots, m\right\}$ be a set of matrices and $l \geq 1$. Then

$$
\rho\left(A_{1}^{\otimes l}, \ldots, A_{m}^{\otimes l}\right)=\rho^{l}\left(A_{1}, \ldots, A_{m}\right) .
$$

Proof. Let $\sigma \in\{1, \ldots, m\}^{l}$. By using the equalities (3) and (5) of Lemma 3] we obtain

$$
\left\|A_{\sigma_{l}}^{\otimes l} \cdots A_{\sigma_{1}}^{\otimes l}\right\|=\left\|A_{\sigma}^{\otimes l}\right\|=\left\|A_{\sigma}\right\|^{l} .
$$

The result then follows from the definition of the joint spectral radius (1.2).

We now would like to combine Theorem 2 with Theorem 1 in order to obtain approximations of arbitrary high accuracy for matrices leaving a cone invariant. In order to do this we need to consider Kronecker products of cones. For two sets $Q_{1} \subseteq R^{n}$ and $Q_{2} \subseteq R^{m}$ let us denote

$$
Q_{1} \otimes Q_{2}=\left\{z=u \otimes v: u \in Q_{1}, v \in Q_{2}\right\}
$$


(In this definition, the vectors $u$ and $v$ are treated as column matrices.) If $K_{1}$ and $K_{2}$ are proper cones then $K_{1} \otimes K_{2}$ does not need to be a proper cone. Indeed, consider for example the proper cones $K_{1}=K_{2}=\mathbf{R}_{+}^{2}$ for which it is easy to see that $K_{1} \otimes K_{2}$ has empty interior in $\mathbf{R}^{4}$. We do however have the following result.

Lemma 4. Let $K_{1}$ and $K_{2}$ be proper cones, then the cone $K=\overline{\operatorname{Conv}\left(K_{1} \otimes K_{2}\right)}$ is a proper cone also.

Proof. The cone $K$ is closed and convex by definition. Assume that int $K=\emptyset$. Then there exists $A \in R^{n m} \backslash\{0\}$ such that

$$
\langle A, z\rangle=0 \quad \forall z \in K \supset K_{1} \otimes K_{2} .
$$

Note that the function $f(u, v)=\langle A, u \otimes v\rangle$ is a bilinear form defined on $K_{1} \times K_{2}$. Since $\operatorname{int}\left(K_{1} \times K_{2}\right) \neq \emptyset, f$ can vanish on this set only if $A=0$. That is a contradiction.

Let us prove now that $K$ contains no straight line. Assume that this is not the case. Then $\operatorname{int} K^{*}=\emptyset$, which implies existence of $B \in R^{n m} \backslash\{0\}$ such that

$$
\langle B, w\rangle=0 \quad \forall w \in K^{*} .
$$

Since $\langle u \otimes v, x \otimes y\rangle=\langle u, x\rangle \cdot\langle v, y\rangle$, we have $K_{1}^{*} \otimes K_{2}^{*} \subseteq K^{*}$. Since $K_{1}^{*}$ and $K_{2}^{*}$ have nonempty interior, relation (3.3) is impossible by the first part of the proof.

We are ready to state the main result of this section.

Theorem 3. Let $\left\{A_{i}: i=1, \ldots, m\right\}$ be a set of matrices leaving a proper cone invariant. Then

$$
\frac{1}{m^{1 / k}} \rho^{1 / k}\left(A_{1}^{\otimes k}+\cdots+A_{m}^{\otimes k}\right) \leq \rho\left(A_{1}, \ldots, A_{m}\right) \leq \rho^{1 / k}\left(A_{1}^{\otimes k}+\cdots+A_{m}^{\otimes k}\right) .
$$

In particular, the joint spectral radius is given by

$$
\rho\left(A_{1}, \ldots, A_{m}\right)=\lim _{k \rightarrow \infty} \rho^{1 / k}\left(A_{1}^{\otimes k}+\cdots+A_{m}^{\otimes k}\right) .
$$

Proof. Assume that the matrices leave the proper cone $K$ invariant. If $A K \subseteq K$, then $(A K)^{\otimes k} \subseteq K^{\otimes k}$ and by Lemma 3, $A^{\otimes k} K^{\otimes k} \subseteq K^{\otimes k}$. But then also $A^{\otimes k} \overline{\text { Conv } K^{\otimes k}} \subseteq$ $\overline{\text { Conv } K^{\otimes k}}$. By Lemma 4 the cone $\overline{\text { Conv } K^{\otimes k}}$ is a proper cone. Thus the matrices $A_{i}^{\otimes k}$ leave a proper cone invariant and by Theorem 1 we have

$$
\frac{1}{m} \rho\left(A_{1}^{\otimes k}+\cdots+A_{m}^{\otimes k}\right) \leq \rho\left(A_{1}^{\otimes k}, \ldots, A_{m}^{\otimes k}\right) \leq \rho\left(A_{1}^{\otimes k}+\cdots+A_{m}^{\otimes k}\right) .
$$

In order to conclude it suffices to use the fact, proved in Theorem 2, that

$$
\rho\left(A_{1}^{\otimes k}, \ldots, A_{m}^{\otimes k}\right)=\rho^{k}\left(A_{1}, \ldots, A_{m}\right) .
$$

It is somewhat surprising to notice that the right hand side in (3.4) does not directly involve any mixed product between the matrices.

An approximation of relative accuracy $(1 / m)^{1 / k}$ can thus be obtained by computing the spectral radius of a single matrix of dimension $n^{k}$. For pairs of matrices some of the relative accuracies and the corresponding matrix sizes are as follows: 


$$
\begin{array}{lllll}
\text { Relative accuracy } & 0.707 & 0.840 & 0.917 & 0.957 \\
\text { Matrix size } & n^{2} & n^{4} & n^{8} & n^{16}
\end{array}
$$

\section{SEmi-Definite LIFTING}

The result in Theorem 3 provides an easy way to evaluate with arbitrary accuracy the joint spectral radius of matrices that leave a proper cone invariant. Unfortunately, matrices do not always leave a proper cone invariant. We show in this section how an invariant cone can always be created by semi-definite lifting.

Let $A \in \mathbf{R}^{n \times n}$ and consider the following linear operator:

$$
X \rightarrow A X A^{T}: \mathbf{R}^{n \times n} \rightarrow \mathbf{R}^{n \times n} .
$$

A matrix representation for this linear operator can be obtained by using the matrix-tovector operator that develops a matrix into a vector by taking its columns one by one. This operator, denoted vec, satisfies the elementary property vec $(C X D)=\left(D^{T} \otimes C\right) \operatorname{vec}(X)$ (see Lemma 4.3.1 in [23]). We therefore have $\operatorname{vec}\left(A X A^{T}\right)=(A \otimes A) \operatorname{vec} X$ and a matrix representation of the linear operator (4.1) is thus simply given by $A \otimes A$.

Consider now the space of symmetric matrices $\mathbf{S}$. This space is a subspace of $\mathbf{R}^{n \times n}$ of dimension $n(n+1) / 2$ and the operator

$$
X \rightarrow A X A^{T}: \mathbf{S} \rightarrow \mathbf{S} .
$$

is again a linear operator. Matrix representations of this operator are of course different from those of the operator (4.1) since, in particular, the spaces on which these operators operate have different dimensions (we describe in the next section how to construct a matrix representations for the operator on symmetric matrices). In the next theorem we prove that even though the operators $X \rightarrow A X A^{T}$ on $\mathbf{S}$ and on $\mathbf{R}^{n \times n}$ are different, their joint spectral radius are equal.

Theorem 4. Let $\left\{A_{i} \in \mathbf{R}^{n \times n}: i=1, \ldots, m\right\}$ and denote by $M_{A}$ a matrix representation of the linear operator $X \rightarrow A X A^{T}: \mathbf{S} \rightarrow \mathbf{S}$. Then we have

$$
\rho\left(M_{A_{1}}, \ldots, M_{A_{m}}\right)=\rho\left(A_{1} \otimes A_{1}, \ldots, A_{m} \otimes A_{m}\right)=\rho^{2}\left(A_{1}, \ldots, A_{m}\right) .
$$

Proof. The second equality is already proved in Theorem 2, we only need to prove the first equality. Let $\|\cdot\|_{F}$ denote the Frobenius matrix norm (i.e., the sum of squares of all entries) and consider the resulting induced operator norms for $X \rightarrow A X A^{T}$ on $\mathbf{R}^{n \times n}$ and S:

$$
\sup _{X \in \mathbf{R}^{n \times n},\|X\|_{F}=1}\left\|A X A^{T}\right\|_{F} \quad \text { and } \sup _{X \in \mathbf{S},\|X\|_{F}=1}\left\|A X A^{T}\right\|_{F} .
$$

We claim that these two operator norms are equal and that the supremum is achieved for some symmetric matrix of rank one. Indeed, using the matrix-to-vector operator vec 
and denoting by $\|\cdot\|$ the usual vector Euclidean norm we get

$$
\begin{aligned}
\sup _{X \in \mathbf{R}^{n \times n},\|X\|_{F}=1}\left\|A X A^{T}\right\|_{F}^{2} & =\sup _{x \in \mathbf{R}^{n^{2}},\|x\|=1}\|(A \otimes A) x\|^{2} \\
& =\sup _{x \in \mathbf{R}^{n^{2}}, x^{T} x=1} x^{T}(A \otimes A)^{T}(A \otimes A) x \\
& =\sup _{x \in \mathbf{R}^{n^{2}}, x^{T} x=1} x^{T}\left(A^{T} A \otimes A^{T} A\right) x
\end{aligned}
$$

For deriving the last equality, we have used (2) in Lemma 3. The supremum in the last expression is achieved by any eigenvector $x$ associated to an eigenvalue of largest magnitude of $A^{T} A \otimes A^{T} A$. Let $\left(\lambda_{i}, v_{i}\right) i=1, \ldots, n$ be the set of eigenvalues/eigenvectors pairs associated to the symmetric matrix $A^{T} A$ and let $\lambda_{1}$ be such that $\left|\lambda_{1}\right| \geq \lambda_{i}$ for all $i$. The eigenvalues/eigenvectors pairs of the matrix $A^{T} A \otimes A^{T} A$ are then given by $\left(\lambda_{i} \lambda_{j}, v_{i} \otimes v_{j}\right)$ for $i, j=1, \ldots, n$. The vector $v_{1} \otimes v_{1}$ is thus an eigenvector of $A^{T} A \otimes A^{T} A$ of largest eigenvalue magnitude. Since $\operatorname{vec}\left(v_{k} v_{k}^{T}\right)=v_{k} \otimes v_{k}$ we see that the supremum of $\left\|A X A^{T}\right\|_{F}$ is achieved for the symmetric rank one matrix $v_{1} v_{1}^{T}$. From this we conclude that

$$
\sup _{X \in \mathbf{R}^{n \times n},\|X\|_{F}=1}\left\|A X A^{T}\right\|_{F}=\sup _{X \in \mathbf{S},\|X\|_{F}=1}\left\|A X A^{T}\right\|_{F}
$$

and thus also

$$
\sup _{\|x\|=1}\|(A \otimes A) x\|=\sup _{\|x\|=1}\left\|\left(M_{A}\right) x\right\| .
$$

Notice that $M_{A_{\sigma}}=\left(M_{A_{i}}\right)_{\sigma}$ and so

$$
\left\|\left(M_{A_{i}}\right)_{\sigma}\right\|=\left\|M_{A_{\sigma}}\right\|=\left\|A_{\sigma} \otimes A_{\sigma}\right\|=\left\|\left(A_{i} \otimes A_{i}\right)_{\sigma}\right\|
$$

and it then suffices to apply the definition of the joint spectral radius to conclude the proof.

A matrix $A$ is positive semi-definite (which we denote by $A \succeq 0$ ) if $v^{T} A v \geq 0$ for all $v \in \mathbf{R}^{n}$. The set of symmetric positive semi-definite matrices, denoted $\mathbf{S}_{+}$, is a cone. It is not a proper cone of $\mathbf{R}^{n \times n}$ because it has empty interior in $\mathbf{R}^{n \times n}$; but it is a proper cone of the set of symmetric matrices $\mathbf{S}$. If $X \in \mathbf{S}_{+}$then we clearly have $A X A^{T} \in \mathbf{S}_{+}$ and so the operator $X \rightarrow A X A^{T}$ leaves the proper cone $\mathbf{S}_{+}$invariant. Combining this observation with Theorem 3 and Theorem 4 we deduce:

Theorem 5. Let $\left\{A_{i} \in \mathbf{R}^{n \times n}: i=1, \ldots, m\right\}$ and denote by $M_{A}$ a matrix representation of the linear operator $X \rightarrow A X A^{T}: \mathbf{S} \rightarrow \mathbf{S}$. Then

$$
\frac{1}{\sqrt{m}} \rho^{1 / 2}\left(M_{A_{1}}+\ldots+M_{A_{m}}\right) \leq \rho\left(A_{1}, \ldots, A_{m}\right) \leq \rho^{1 / 2}\left(M_{A_{1}}+\ldots+M_{A_{m}}\right) .
$$

Note that for $m=2$ the bounds (4.3) are not so bad. Indeed, $1 / \sqrt{2}>0.7$ and so the relative accuracy of the approximation is at least 70\%. The main interest of this result resides however in the possibility of applying it recursively. Starting from an initial matrix 
$A \in \mathbf{R}^{n \times n}$ we define a sequence of operators acting on spaces of increasing dimensions. For $l=1$ we define

$$
A^{[1]}(x) \equiv A x \quad x \in E_{1} \equiv \mathbf{R}^{n}
$$

and for $l \geq 2$ we define the operators recursively

$$
A^{[l]}(x) \equiv M_{A^{[l-1]}}(x) \quad x \in E_{l} \equiv \mathbf{S}\left(E_{l-1}\right) .
$$

Assume for the simplicity of the presentation that $m=2$ and let $A_{1}, A_{2} \in \mathbf{R}^{n \times n}$. From Theorem 4 we know that

$$
\rho\left(A_{1}^{[l]}, A_{2}^{[l]}\right)=\rho^{2^{l}}\left(A_{1}, A_{2}\right)
$$

On the other hand, for any $l \geq 1$ the operators $A_{1}^{[l]}$ and $A_{2}^{[l]}$ leave the cone $\mathbf{S}_{+}\left(E_{l-1}\right)$ invariant. Therefore, in view of Theorem 1, we have

$$
\frac{1}{2} \rho\left(A_{1}^{[l]}+A_{2}^{[l]}\right) \leq \rho\left(A_{1}^{[l]}, A_{2}^{[l]}\right) \leq \rho\left(A_{1}^{[l]}+A_{2}^{[l]}\right) .
$$

Combining these last two expressions, we get the following bounds:

$$
\left(\frac{1}{2}\right)^{1 / 2^{l}}\left[\rho\left(A_{1}^{[l]}+A_{2}^{[l]}\right)\right]^{1 / 2^{l}} \leq \rho\left(A_{1}, A_{2}\right) \leq\left[\rho\left(A_{1}^{[l]}+A_{2}^{[l]}\right)\right]^{1 / 2^{l}} .
$$

Note that

$$
1-\frac{\ln 2}{2^{l}} \leq\left(\frac{1}{2}\right)^{1 / 2^{l}} \leq 1
$$

and thus the improvement in the quality of our approximation is quite fast. Unfortunately, the dimensions of the spaces $E_{l}$ are also growing fast; we have

$$
n_{l+1}=\frac{1}{2} n_{l}\left(n_{l}+1\right)
$$

(where $n_{i}=\operatorname{dim} E_{i}$ ) and therefore asymptotically we have $n_{l}=O\left((n / 2)^{2^{l}}\right)$. Let us display, for a pair of matrices, the relative accuracy of our approximation as a function of the resulting dimension.

\begin{tabular}{|r|r|r|r|r|}
\hline Steps & Accuracy & $n=2$ & $n=10$ & $n=100$ \\
\hline 1 & 0.707 & 3 & 55 & 5050 \\
2 & 0.840 & 6 & 1540 & $*$ \\
3 & 0.917 & 21 & 118570 & $*$ \\
4 & 0.957 & 231 & $*$ & $*$ \\
5 & 0.978 & 26796 & $*$ & $*$ \\
\hline
\end{tabular}

We use the symbol $*$ to mark the cases for which the dimension of the auxiliary problem goes beyond the abilities of modern computers. 


\section{NumericAl IMPLEMENTATION AND EXAMPLES}

The recursive semi-definite lifting for obtaining approximations of the joint spectral radius of increasing accuracy may be difficult to implement because the matrix $M_{A}$ is not easy to express in terms of the matrix $A$. Consider for example the case of $2 \times 2$ matrices. Let

$$
A=\left(\begin{array}{ll}
a_{11} & a_{12} \\
a_{21} & a_{22}
\end{array}\right)
$$

then one easily compute

$$
M_{A}=\left(\begin{array}{ccc}
a_{11}^{2} & 2 a_{11} a_{12} & a_{12}^{2} \\
a_{11} a_{21} & a_{11} a_{22}+a_{12} a_{21} & a_{12} a_{22} \\
a_{21}^{2} & 2 a_{21} a_{22} & a_{22}^{2}
\end{array}\right) .
$$

In general, the matrix $M_{A}$ can be expressed as follows:

$$
M_{A}=\left(Q^{T} Q\right)^{-1} Q^{T} P^{-1}(A \otimes A) P Q
$$

where $P$ is a particular permutation matrix of size $n^{2}$ and $Q$ is given by

$$
Q=\left(\begin{array}{cc}
I_{n} & 0 \\
0 & I_{\frac{n(n-1)}{2}} \\
0 & I_{\frac{n(n-1)}{2}}
\end{array}\right)
$$

(the matrix $I_{k}$ is the identity matrix of size $k$ ). One difficulty with the expression (5.1) is that the permutation matrix $P$ is tedious to construct; it essentially relates the column decomposition of a matrix with its diagonal decomposition. The permutation matrix can of course be constructed but the implementation of this construction is somewhat cumbersome. A way to bypass this difficulty can be achieved by using the semi-definite lifting only once, and then apply only Kronecker liftings. The semi-definite lifting can be performed either at the beginning or at the end of the process, leading to the bounds

$$
\frac{1}{m} \rho\left(M_{A_{1}}^{\otimes l}+\cdots+M_{A_{m}}^{\otimes l}\right) \leq \rho^{2 l}\left(A_{1}, \ldots, A_{m}\right) \leq \rho\left(M_{A_{1}}^{\otimes l}+\cdots+M_{A_{m}}^{\otimes l}\right)
$$

and

$$
\frac{1}{m} \rho\left(M_{A_{1}^{\otimes l}}+\cdots+M_{A_{m}^{\otimes l}}\right) \leq \rho^{2 l}\left(A_{1}, \ldots, A_{m}\right) \leq \rho\left(M_{A_{1}^{\otimes l}}+\cdots+M_{A_{m}^{\otimes l}}\right) .
$$

The validity of these inequalities results from the combination of Theorem 1 , Theorem 2. Lemma 4 and Theorem 4 The expressions (5.2) and (5.3) both provide a relative accuracy of $(1 / m)^{1 /(2 l)}$ but involve matrices of different size. The matrices $M_{A_{i}}^{\otimes l}$ have size $(n(n+1) / 2)^{l}$ whereas the matrices $M_{A_{i}^{\otimes l}}$ have size $n^{l}\left(n^{l}+1\right) / 2>(n(n+1) / 2)^{l}$. In addition to this, the matrices $M_{A_{i}}^{\otimes l}$ are easier to compute than $M_{A_{i}^{\otimes l}}$ since they necessitate the evaluation of semi-definite liftings of smaller matrices.

Of course, other combinations of Kronecker and semi-definite liftings are also possible. Whenever a $k$-th Kronecker power is used, the current relative accuracy is $k$-th rooted, and whenever a semi-definite lifting is used, the relative accuracy is square rooted. Moreover, 
at least one semi-definite lifting is needed if the original matrices do not leave a proper cone invariant.

For the numerical implementation of these approximations notice also that the spectral radius of $M_{A_{1}}+\cdots+M_{A_{m}}$ is the spectral radius of the linear operator

$$
B: X \rightarrow A_{1} X A_{1}^{T}+\cdots+A_{m} X A_{m}^{T}: \mathbf{S} \rightarrow \mathbf{S}
$$

and it can therefore be found by standard linear algebra techniques, or by solving the following linear inequality optimization problem

$$
\inf _{X, \tau}\left\{\tau: \tau X \succeq A_{1} X A_{1}^{T}+\cdots+A_{m} X A_{2}^{T}, X \succ 0\right\}
$$

for which efficient convex optimization algorithms are available (see, e.g., [12]) that are implemented in standard commercial softwares such as the Matlab "LMI Control Toolbox".

\section{Computational COMplexity analysis}

The joint spectral radius can be approximated to arbitrary accuracy. It is proved in 32 that, unless $\mathrm{P}=\mathrm{NP}$, approximating algorithms of relative accuracy $1-\epsilon$ for pairs of matrices cannot run in time polynomial in the size of the matrices and in $\ln (1 / \epsilon)$. It was later noticed (see the note 9 on page 1260 of [9]) that a careful examination of the proof in 32 shows that, unless $\mathrm{P}=\mathrm{NP}$, there are no approximation algorithm of relative accuracy $1-\epsilon$ that run in time polynomial in the size of the matrices and in $1 / \epsilon$. This is however not proved in [9]. In this section we prove this result by extracting the essential part of the proof in 32 .

We proceed by reduction from the classical NP-complete satisfiability problem 3-SAT. This problem is defined as follows. Consider a set $\left\{x_{1}, \ldots x_{n}\right\}$ of Boolean variables. A literal is either a Boolean variable $x_{i}$, or its negation $\bar{x}_{i}$. A three-literal clause is a disjunction of three literals (e.g., $x_{3} \vee \bar{x}_{5} \vee \bar{x}_{6}$ ). The 3 -SAT problem is the problem of determining, for a given collection of three-literal clauses, if there exists a truth assignment for the variables that simultaneously satisfies all clauses. This problem is known to be NP-complete; finding a polynomial time algorithm for the problem is exactly equivalent to proving that $\mathrm{P}=\mathrm{NP}$.

The complexity result proved in 32 is based on the construction of a pair of matrices which we briefly outline (the complete construction can easily be extracted from [32]). From a given instance of 3-SAT with $n$ variables and $p$ clauses two square matrices $A_{1}, A_{2}$ are constructed in polynomial time. The matrices have their entries in $\{0,1\}$, are of size $(n+1)(p+1)$, and have a joint spectral radius that satisfies:

$$
\begin{array}{ll}
\rho\left(A_{1}, A_{2}\right) \geq p^{1 /(n+2)} & \text { if the instance is satisfiable } \\
\rho\left(A_{1}, A_{2}\right) \leq(p-1)^{1 /(n+2)} & \text { if the instance is not satisfiable. }
\end{array}
$$


Any algorithm of relative accuracy $(1-1 / p)^{1 /(n+2)}$ allows to make the distinction between these two cases. Moreover, since

$$
\left(1-\frac{1}{p}\right)^{1 /(n+2)} \leq 1-\frac{1}{p(n+2)} \leq 1
$$

it is clear that an approximation algorithm of relative accuracy $1-1 / p(n+2)$ also allows to decide 3-SAT. Since the size of the matrices in the construction are given by $(n+1)(p+1)$, we deduce:

Theorem 6. Unless $P=N P$, the problem of approximating the joint spectral radius of two square matrices with $\{0,1\}$ entries and with relative accuracy $1-\epsilon$ cannot be obtained in time polynomial in the size of the matrices and in $1 / \epsilon$.

From Theorem 3 we know that the spectral radius of two matrices with nonnegative entries is given by $\rho\left(A_{1}, A_{2}\right)=\lim _{k \rightarrow \infty} \rho^{1 / k}\left(A_{1}^{\otimes k}+A_{2}^{\otimes k}\right)$ and so the quantity $\lim _{k \rightarrow \infty} \rho^{1 / k}\left(A_{1}^{\otimes k}+\right.$ $\left.A_{2}^{\otimes k}\right)$ is NP-hard to approximate in the sense given in Theorem 6. Theorem 3 provides also a rate of convergence for the approximations $\rho^{1 / k}\left(A_{1}^{\otimes k}+A_{2}^{\otimes k}\right)$. From this we may prove the following complexity result.

Theorem 7. The problem of determining, for a given integer $k \geq 0$ and for a given pair of matrices $A_{1}, A_{2}$ with nonnegative entries, if

$$
\rho\left(A_{1}^{\otimes k}+A_{2}^{\otimes k}\right)<1
$$

is a problem that is NP-hard.

Proof. We proceed by reduction from 3-SAT. From a given instance of 3-SAT with $n$ variables and $p$ clauses we use the construction given in 32 to construct two square matrices $B_{1}, B_{2}$ that have their entries in $\{0,1\}$, are of size $(n+1)(p+1)$, and have a joint spectral radius that satisfies:

$$
\begin{array}{ll}
\rho\left(B_{1}, B_{2}\right) \geq p^{1 /(n+2)} & \text { if the instance is satisfiable } \\
\rho\left(B_{1}, B_{2}\right) \leq(p-1)^{1 /(n+2)} & \text { if the instance is not satisfiable. }
\end{array}
$$

We then choose $\alpha \in \mathbf{R}$ with $0<\alpha<1, r \in \mathbf{Q}$ and $k \geq 0$ such that $\alpha<(1 / 2)^{1 / k}$ and

$$
(p-1)^{\frac{1}{n+2}}<\frac{1}{\alpha}(p-1)^{\frac{1}{n+2}}<r<\alpha q^{\frac{1}{n+2}}<p^{\frac{1}{n+2}} .
$$

Consider now the matrices $A_{1}=(1 / r) B_{1}$ and $A_{2}=(1 / r) B_{2}$. We claim that $\rho\left(A_{1}^{\otimes k}+\right.$ $\left.A_{2}^{\otimes k}\right)<1$ iff the instance of 3 -SAT is not satisfiable. Indeed, assume first that the instance of 3-SAT is not satisfiable. Then

$$
\rho\left(B_{1}, B_{2}\right) \leq(p-1)^{1 /(n+2)} .
$$

Since $\rho\left(A_{1}^{\otimes k}+A_{2}^{\otimes k}\right)$ is an approximation of relative accuracy $1 / 2^{2 k}$ of $\rho\left(A_{1}, A_{2}\right)$, we have

$$
\rho\left(A_{1}^{\otimes k}+A_{2}^{\otimes k}\right) \leq 2^{2 k} \rho\left(A_{1}, A_{2}\right) \leq \frac{2^{2 k}}{r} \rho\left(B_{1}, B_{2}\right) \leq \frac{2^{2 k}}{r}(p-1)^{1 /(n+2)}
$$


and by using the inequalities (6.1) we conclude $\rho\left(A_{1}^{\otimes k}+A_{2}^{\otimes k}\right)<1$. In an analogous way one see that, if the instance of 3-SAT is satisfiable, then $\rho\left(A_{1}^{\otimes k}+A_{2}^{\otimes k}\right)>1$ and the proof is therefore complete.

\section{ELLIPSOID APPROXIMATION}

Let us now compare our approximation of the joint spectral radius with another approximation appearing in the literature. The following approximation, called ellipsoid approximation, is introduced in [6]:

$$
\hat{\rho}\left(A_{1}, \ldots, A_{m}\right)=\left[\inf _{X, \tau}\left\{\tau: \tau X \succeq A_{i} X A_{i}^{T}, i=1, \ldots, m, X \succ 0\right\}\right]^{1 / 2} .
$$

This expression corresponds to the best ellipsoid norm for the set of matrices. In [6] the following inequalities are proved

$$
\frac{1}{\sqrt{n}} \hat{\rho}\left(A_{1}, \ldots, A_{m}\right) \leq \rho\left(A_{1}, \ldots, A_{m}\right) \leq \hat{\rho}\left(A_{1}, \ldots, A_{m}\right) .
$$

Thus, the ellipsoid approximation has a relative accuracy of $1 / \sqrt{n}$. This accuracy decreases with the size of the matrices but does not depend on the number of matrices in the set. Using our results we can prove that the relative accuracy of the ellipsoid approximation is in fact bounded by $1 / \sqrt{m}$ where $m$ is the number of matrices.

Theorem 8. Let $\left\{A_{i}: i=1, \ldots, m\right\}$ be a set of matrices and define the ellipsoid approximation $\hat{\rho}$ by (7.1). Then

$$
\frac{1}{\sqrt{m}} \hat{\rho}\left(A_{1}, \ldots, A_{m}\right) \leq \rho\left(A_{1}, \ldots, A_{m}\right) \leq \hat{\rho}\left(A_{1}, \ldots, A_{m}\right) .
$$

Proof. It is easy to see that $\rho\left(A_{1}, \ldots, A_{m}\right) \leq \hat{\rho}\left(A_{1}, \ldots, A_{m}\right)$. For proving the first inequality, note that the spectral radius of the linear operator

$$
B: X \rightarrow \sum_{i=1}^{m} A_{i} X A_{i}^{T}: \quad \mathbf{S} \rightarrow \mathbf{S}
$$

can be represented as follows:

$$
\rho(B)=\inf _{X, \tau}\left\{\tau: \tau X \succeq \sum_{i=1}^{m} A_{i} X A_{i}^{T}, X \succ 0\right\} .
$$

If a pair $(X, \tau)$ is feasible for the optimization problem in (17.2), then it is also feasible for the optimization problem in (7.1). Therefore

$$
\hat{\rho}\left(A_{1}, \ldots, A_{m}\right) \leq \rho^{1 / 2}(B) .
$$

Finally, by Theorem 5 we have

$$
\frac{1}{\sqrt{m}} \rho^{1 / 2}(B) \leq \rho\left(A_{1}, \ldots, A_{m}\right)
$$


and so we get the following bounds

$$
\frac{1}{\sqrt{m}} \hat{\rho}\left(A_{1}, \ldots, A_{m}\right) \leq \frac{1}{\sqrt{m}} \rho^{1 / 2}(B) \leq \rho\left(A_{1}, \ldots, A_{m}\right) \leq \hat{\rho}\left(A_{1}, \ldots, A_{m}\right) \leq \rho^{1 / 2}(B) .
$$

Thus we see that the ellipsoid estimate $\hat{\rho}\left(A_{1}, \ldots, A_{m}\right)$ has relative quality $1 / \sqrt{m}$.

By using Theorem 2 one can obtain a statement analogous to that of Theorem 3.

Corollary 3. Let $\left\{A_{i}: i=1, \ldots, m\right\}$ be a set of matrices and define the ellipsoid approximation $\hat{\rho}$ by (7.1). Then

$$
\frac{1}{m^{1 /(2 k)}} \hat{\rho}^{1 / k}\left(A_{1}^{k}, \ldots, A_{m}^{k}\right) \leq \rho\left(A_{1}, \ldots, A_{m}\right) \leq \hat{\rho}^{1 / k}\left(A_{1}^{k}, \ldots, A_{m}^{k}\right) .
$$

For $k-1$, this result is also proved in [1]. For arbitrary $k$ a proof can be reconstructed from the result proved in 21. Notice however that approximation algorithms that are directly based on (17.4) are not efficient because they require to solve linear matrix inequalities of very large dimension. Approximations based on (5.2) are easier to obtain because there exists many efficient numerical algorithms for approximating the spectral radius of a matrix.

\section{Discussion}

The results presented in this paper introduce the possibility to compute approximations for the joint spectral radius with worst-case theoretical guarantees. Of course, the computational complexity of these estimates needs further examination. Indeed, the spectral structure of the matrix $A^{\otimes k}$ is very simple and this simplicity must be inherited somehow by the matrix $A_{1}^{\otimes k}+A_{2}^{\otimes k}$. It therefore appears to be an interesting problem to investigate the possibility of constructing efficient procedures for finding the spectral radius of this sum; of course there are theoretical limitations on what can be achieved since we have shown that the problem of deciding $\rho\left(A_{1}^{\otimes k}+A_{2}^{\otimes k}\right) \leq 1$ is NP-hard.

Another interesting issue is that of determining if worst-case theoretical guarantees can be provided for the approximation

$$
\max _{\sigma \in\{1, \ldots, m\}^{k}} \rho\left(A_{\sigma}\right)^{1 / k} \leq \rho\left(A_{1}, \ldots, A_{m}\right) .
$$

In many numerical examples, this approximation does in fact perform at least as well.

\section{REFERENCES}

[1] T. Ando, M. Shih. Simultaneous Contractibility, SIAM Journal on Matrix Analysis and Applications Volume 19, Number 2 pp. 487-498, 1998.

[2] N. E. Barabanov. Lyapunov indicators of discrete inclusions, parts I, II and III, Avtomatika i Telemekhanika, 2 , 40-46, 3, 24-29 and 5, 17-24, 1988. Translation in Automat. Remote Control part I, 49, no. 3, 152-157, part II, 49, no. 5, 558-565, 1988.

[3] M. A. Berger and Y. Wang, Bounded Semigroups of Matrices, Journal of Linear Algebra and its Applications, vol.166, 1992, pp.21-27. 
[4] V. D. Blondel, J. Theys and A. A. Vladimirov, An elementary counterexample to the finiteness conjecture, SIAM Journal on Matrix Analysis, 24:4, pp. 963-970, 2003.

[5] V. D. Blondel and Yu. Nesterov. Fast and precise approximations of the joint spectral radius. CORE discussion paper, number 2003/97, available from http://www.core.ucl.ac.be/services/COREdp03.html

[6] V. D. Blondel, Yu. Nesterov, J. Theys, Polynomial-time approximation algorithms for the joint spectral radius, submitted.

[7] V. D. Blondel, Yu. Nesterov, J. Theys, Approximations of the rate of growth of switched linear systems, To appear in the proceedings of HSSC 2003, Lecture Notes in Computer Science, Springer Verlag, 2003.

[8] V. D. Blondel and J. N. Tsitsiklis. The boundedness of all products of a pair of matrices is undecidable. Systems and Control Letters, 41:2, pp. 135-140, 2000.

[9] V. D. Blondel and J. N. Tsitsiklis. A survey of computational complexity results in systems and control. Automatica, 36:9, pp. 1249-1274, 2000.

[10] V. D. Blondel, S. Gaubert, and J. N. Tsitsiklis, Approximating the spectral radius of sets of matrices in the max-algebra is NP-hard, IEEE Transactions on Automatic Control, 45:9, pp. 1762-1765, 2000.

[11] T. Bousch and J. Mairesse. Asymptotic height optimization for topical IFS, Tetris heaps and the finiteness conjecture. J. Amer. Math. Soc., 15, pp. 77-111, 2002.

[12] S. Boyd, L. El Ghaoui, E. Feron, and V. Balakrishnan, Linear Matrix Inequalities in System and Control Theory, SIAM, 1994.

[13] Brayton, R. and C. Tong (1980). Constructive stability and asymptotic stability of dynamical systems, IEEE Transactions on Circuits and Systems, 27, 1121-1130.

[14] I. Daubechies and J. C. Lagarias. Sets of matrices all infinite products of which converge. Linear Algebra Appl., 161, pp. 227-263, 1992.

[15] I. Daubechies and J. C. Lagarias. Corrigendum/addendum to: Sets of matrices all infinite products of which converge. Linear Algebra Appl., 327, pp. 69-83, 2001.

[16] A. A. Vladimirov, L. Elsner, and W.-J. Beyn. Stability and paracontractivity of discrete linear inclusions. Linear Algebra Appl., 312, pp. 125-134, 2000.

[17] G. Gripenberg, Computing the joint spectral radius, Linear Algebra Appl. 234, 43-60, 1996.

[18] N. Guglielmi, M. Zennaro. On the zero-stability of variable stepsize multistep methods: the spectral radius approach, Numer. Math., 88, 445-458, 2001.

[19] L. Gurvits. Stability of discrete linear inclusions. Linear Algebra Appl., 231, pp. 47-85, 1995.

[20] L. Gurvits. Stability and observability of discrete linear inclusion - Finite automata approach. Book of abstracts, International Symposium on the Mathematical Theory of Networks and Systems, Kobe, Japan, pp. 166-167, 1991.

[21] L. Gurvits. Stabilities and Controllabilities of Switched Systems (with Applications to the Quantum Systems). Proceedings of the Fifteenth International Symposium on Mathematical Theory of Networks and Systems, University of Notre Dame, IN, USA, 2002

[22] R.A. Horn and C.R. Johnson. Topics in Matrix Analysys. Cambrige University Press, 1991.

[23] R. A. Horn and C. R. Johnson. Topics in Matrix Analysis. Cambridge Univ. Press, Cambridge, 1991.

[24] V. S. Kozyakin. Algebraic unsolvability of problem of absolute stability of desynchronized systems. Automation and Remote Control, 51, pp. 754-759, 1990.

[25] J.C. Lagarias and Y. Wang, The finiteness conjecture for the generalized spectral radius of a set of matrices, Linear Algebra Appl., 214, 17-42, 1995.

[26] D. Liberzon, J. P. Hespanha and A. S. Morse, Stability of switched systems: a Lie-algebraic condition, Systems ans Control Letters, volume 37, 3, 1999,pp.117-122.

[27] M. Maesumi, An Efficient Lower Bound for the Generalized Spectral Radius, Linear Algebra and its Applications, 240, pp.1-7, 1996. 
[28] B.E. Moision, A. Orlitsky, P.H. Siegel, On Codes That Avoid Specified Differences, IEEE Trans. Inform. Theory, vol. 47, no. 1, pp. 433-442, January 2001.

[29] G.-C. Rota and W. G. Strang. A note on the joint spectral radius. Indag. Math., 22, pp. 379-381, 1960.

[30] R. T. Rockafellar, Convex Analysis. Princeton, NJ: Princeton University Press, 1970.

[31] G. Strang. The joint spectral radius. Commentary by Gilbert Strang on paper number 5. In "Collected works of Gian-Carlo Rota", 2001.

[32] J. N. Tsitsiklis, V. D. Blondel The Lyapunov exponent and joint spectral radius of pairs of matrices are hard - when not impossible - to compute and to approximate. Mathematics of Control, Signals, and Systems, 10, pp. 31-40, 1997.

[33] J. N. Tsitsiklis, On the stability of asynchronous iterative processes, Math. Systems Theory, 20 , 137-153, 1987.

[34] J.N. Tsitsiklis, The Stability of the Products of a Finite Set of Matrices, Open Problems in Communication and Computation, T.M. Cover and B. Gopinath (Eds.), Springer-Verlag, New York, 161-163, 1987.

[35] A. A. Vladimirov, L. Elsner, and W.-J. Beyn. Stability and paracontractivity of discrete linear inclusions. Linear Algebra Appl., 312, pp. 125-134, 2000.

[36] F. Wirth. The generalized spectral radius and extremal norms. Linear Algebra Appl., 342, pp. 17-40, 2002.

[37] F. Wirth. On the calculation of real time-varying stability radii. International Journal of Robust and Nonlinear Control, 8:1043-1058, 1998.

[38] T. Yu, Bin Han and Michael Overton, Design of Hermite Subdivision Schemes aided by Spectral Radius Optimization. Preprint.

Division of Applied Mathematics, Université Catholique de Louvain, 4 avenue Georges

Lemaitre, B-1348 Louvain-LA-Neuve, Belgium

E-mail address: blondel@inma.ucl.ac.be

Center for Operations Research and Econometrics (CORE), Catholic University of Louvain (UCL), 34 voie du Roman Pays, 1348 Louvain-la-Neuve, Belgium

E-mail address: nesterov@core.ucl.ac.be 